\title{
Effect of mutual coupling on torque production in switched reluctance motors
}

\author{
Jill A. Walker, ${ }^{\text {a) }}$ David G. Dorrell, and Calum Cossar \\ SPEED Laboratory, Department of Electronics and Electrical Engineering, University of Glasgow, Rankine \\ Building, Oakfield Avenue, Glasgow, Lanarkshire G12 8LT, United Kingdom
}

\begin{abstract}
In many cases, the normal operation of switched reluctance machines requires excitation of two or more phases simultaneously. When multiple phases are conducting simultaneously, the flux paths from each phase will overlap, which may lead to localized saturation. In such cases, the flux linkage must be considered a function not just of the current in the test winding but of all excited windings. The degree of mutual coupling between phases influences the per-phase magnetization curves and torque characteristics. In machines with even phase numbers, the degree of mutual coupling between phases varies due to discontinuities in the phase polarity arrangement. From nonlinear finite element simulations, it is possible to compare the $i-\psi$ loop diagrams under single-phase and multiphase excitations, and hence the torque produced. The mutual flux linkage from each phase can be calculated separately for each rotor position using the frozen permeability method, to further analyze the mutual coupling effects. For a given excitation current profile, the torque can be maximized by careful arrangement of the phase polarities.
\end{abstract}

\section{INTRODUCTION}

The magnetization characteristics of the switched reluctance motor are normally represented by per-phase static magnetization curves or $i-\psi$ loops. ${ }^{1}$ The torque produced by each phase can be calculated from the area enclosed by the current/flux linkage or $i-\psi$ trajectory. Such characteristics are normally measured with only one phase excited and so mutual coupling between phases is ignored.

In the switched reluctance motor, the currents in each phase are switched in sequence. In the ideal case, they are square wave, with instantaneous rise and fall times. In reality, it can take several degrees of rotation for the current to rise or fall. This can lead to overlap between adjacent phase currents. In some cases, the switching angle may be advanced to increase torque production; this also results in overlapping current (and flux-linkage) profiles.

When two or more phases are conducting simultaneously, the flux paths from each phase share sections of the laminations, leading to saturation and lower permeabilities in localized regions of the steel, or conversely to reduction in flux density in the steel and increased permeability. When the phases share saturated regions of steel, the phase flux linkage and torque are functions of the currents in all excited phases. In such cases, it is necessary to take mutual coupling between phases into account to accurately predict the magnetization curves or $i-\psi$ loop of each phase.

The degree of mutual coupling is dependent on the polarity arrangement of the phase-in particular, whether the adjacent phases are of the same or opposite polarities. ${ }^{2}$ Figure 1 shows the cross section of an 8/6, four-phase motor with two phases of same polarity conducting. For threequarters of the stator back iron, the fluxes are additive. In

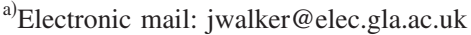

these sections of the back iron, the steel is most likely to saturate. This leads to reduced localized permeabilities and lower flux linkage per phase for a given input current. In the remaining quarter of the back iron, the fluxes flow in opposing directions, so saturation is unlikely. Figure 2 shows the same motor, with adjacent phases of opposite polarities. In this case, there are only additive fluxes for one-quarter of the stator back iron. As such, the case where the phases are of opposite polarities would be expected to show less prominent mutual coupling effects. The degree of mutual coupling can be minimized by careful design of the motor cross section. ${ }^{3}$

\section{FINITE ELEMENT MODELING OF MUTUAL COUPLING}

The effects of mutual coupling can be seen in the shape of the $i-\psi$ trajectory. The $i-\psi$ trajectories can be calculated during normal operation (with each phase being switched in

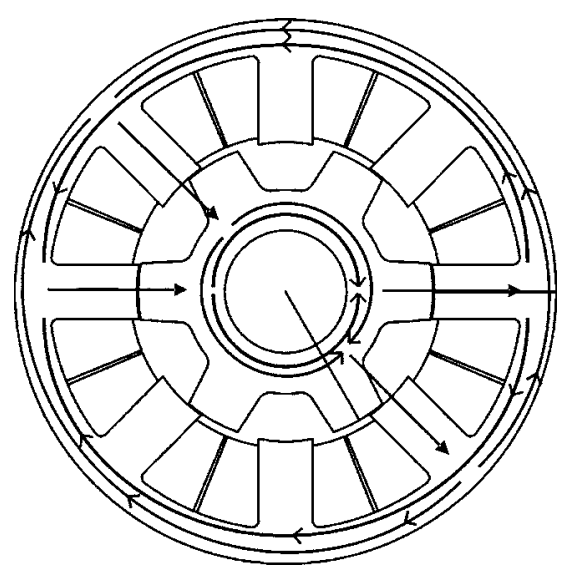

FIG. 1. Flux plots NN polarities. 


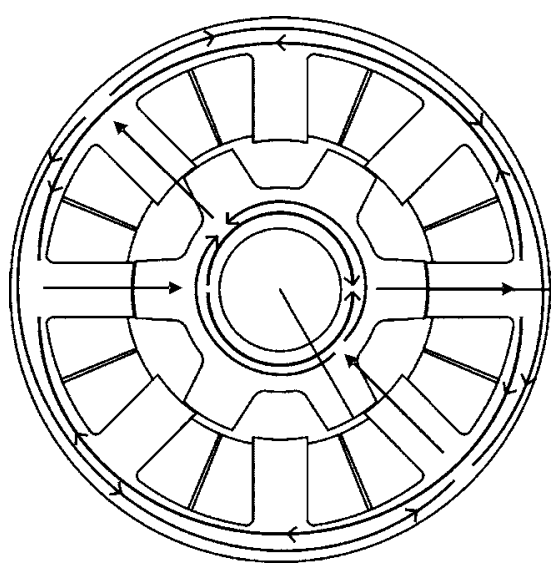

FIG. 2. Flux plots NS polarities.

turn) and for each phase singly excited. Any differences between the resulting $i-\psi$ loops are due to the mutual interaction between the phases. The change in total flux linkage in each phase, due to the other excited phases, is easily determined from nonlinear finite element simulations.

Figure 3 shows the $i-\psi$ loop for one phase of the four phase 8/6 motor for three cases-when the phase is singly excited, when all phases are excited in sequence (phase currents overlap at switching regions) and the polarity of the next phase in the sequence is the same, and when there is current overlap and the polarity of the next phase is opposite. The current trajectories are the same in all three cases (the control system operates in current-control mode with fixed turn-on and turn-off times).

Figure 3 clearly shows that the arrangement of the phase polarities will have a significant effect on the shape of the $i-\psi$ loop and the area enclosed. The area of the $i-\psi$ loop is greater when the adjacent phases are of opposite polarities. In motors with odd phase numbers, this would be the standard configuration. However, for motors with even phase numbers there will always be a discontinuity in the phase polarity arrangement, whereby two adjacent poles must have the same polarity. For example, in the 4 phase test motor, the original winding configuration leads to a N-S-N-S-S-N-S-N

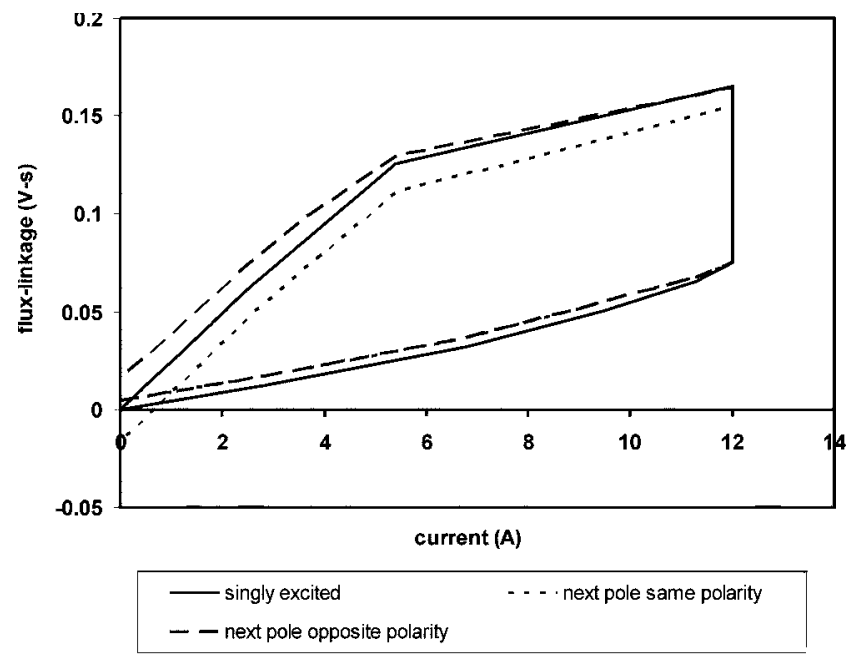

FIG. 3. $i-\psi$ loops for three different cases.
TABLE I. Loop torque for different phase polarity combinations.

\begin{tabular}{cc}
\hline \hline Phase combination (previous/current/next) & Torque produced $(\mathrm{N} \mathrm{m})$ \\
\hline NSS or SNN & 0.6927 \\
NNN or SSS & 0.8321 \\
NSN or SNS & 0.9197 \\
NNS or SSN & 1.0585 \\
\hline \hline
\end{tabular}

pole arrangement (adjacent poles of phases 4 and 1 have same polarity). From nonlinear solutions with all phases excited, the per-phase torque for each combination of phase polarities can be calculated. The per-phase average torque over one cycle, as calculated from the area of the $i-\psi$ loop, is given in Table I for each possible polarity combination.

From Table I, it can be seen that the phase torques will be unbalanced for the same current excitation, due to differences in the pole combinations for each phase. The maximum torque will occur in phase 4 of the test motor, and the minimum torque will occur in phase 1. Phases 2 and 3 produce the same level of torque.

The arrangement of phase polarities also affects the amount of ripple in the complete torque wave form under normal operation. When the two adjacent conducting phases are of the same polarity, the peak instantaneous torque is higher than when the phases are of opposite polarities. The difference in minimum torque between the two cases is much smaller than the difference in peak torque, resulting in a significantly higher torque ripple $(>25 \%)$ for the case when the adjacent phases are of opposite polarities. In any complete cycle of operation, the ripple will vary, due to discontinuity in the phase polarity arrangement.

The effect of phase polarity arrangement on the efficiency of the motor is also significant. The input current wave form is the same regardless of the phase polarity arrangement, so that the copper losses are the same for all cases. There will be a small difference in the iron losses between different polarity arrangements; the iron losses themselves account for only a small percentage of the total losses. As such, the polarity combination that gives the greatest torque (NNS or SSN) will result in a higher efficiency than other polarity combinations.

Although the nonlinear finite element simulations prove that the phase torques are heavily dependent on the winding polarity arrangements, they give no indication as to what proportion of the total flux linkage is due to self-flux-linkage and what proportion is due to mutual flux linkage. The induced mutual flux linkages in all phases can be determined by running frozen permeability finite element simulations for each phase in turn. ${ }^{4,5}$ The mutual, self-, and total flux linkages for phases 1 and 4 (determined from a combination of nonlinear and frozen permeability solutions), for windings polarities of N-S-N-S are given in Figs. 4 and 5, respectively.

Figure 4 shows that the mutual flux linkage from phase 2 seen in phase 1 is positive, creating a positive total flux linkage before phase 1 has been excited. As the rotor changes position, phase 1 itself is excited, which increases the positive flux linkage. As the current in phase 1 is switched off, phase 4 turns on. The mutual flux linkage created by phase 4 


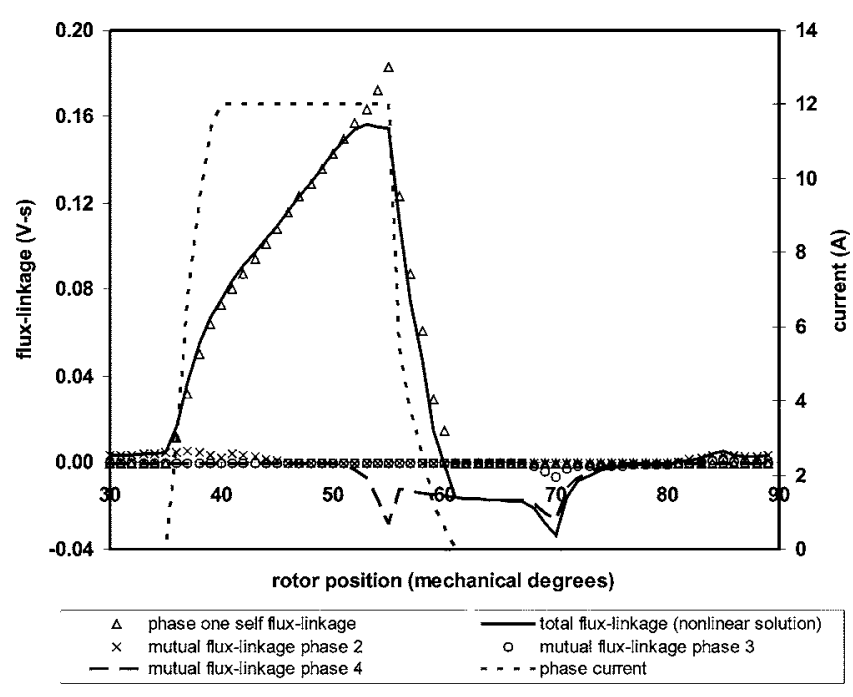

FIG. 4. Flux linkages of phase 1 from finite element solutions.

is negative. As the current in phase 1 decreases, the mutual flux linkage becomes more dominant, until the total flux linkage becomes negative. There is no significant mutual flux linkage from phase 3 , as it is not adjacent to phase 1. Figure 4 clearly illustrates that the cause of the crossover seen in the $i-\psi$ loop is the combination of positive mutual flux linkage from the preceding phase in the sequence and negative mutual flux linkage from the next phase.

Figure 5 shows the self- and mutual flux linkages for phase 4 . The mutual flux linkage from phase 1 is shown to be negative, resulting in a negative total flux linkage at zero current in phase 4 . As the excitation of phase 4 increases, the total flux linkage becomes positive. When the current from phase 4 decreases, the current in phase 3 is introduced, creating a positive mutual flux linkage. This results in a positive total flux linkage for zero current in phase 4. Once again, there is no significant mutual coupling between the remaining nonadjacent phase (phase 2). The combination of negative mutual flux linkage at the beginning and positive mutual flux linkage at the end of the $i-\psi$ loop results in the maximum possible phase torque for the given excitation wave forms.

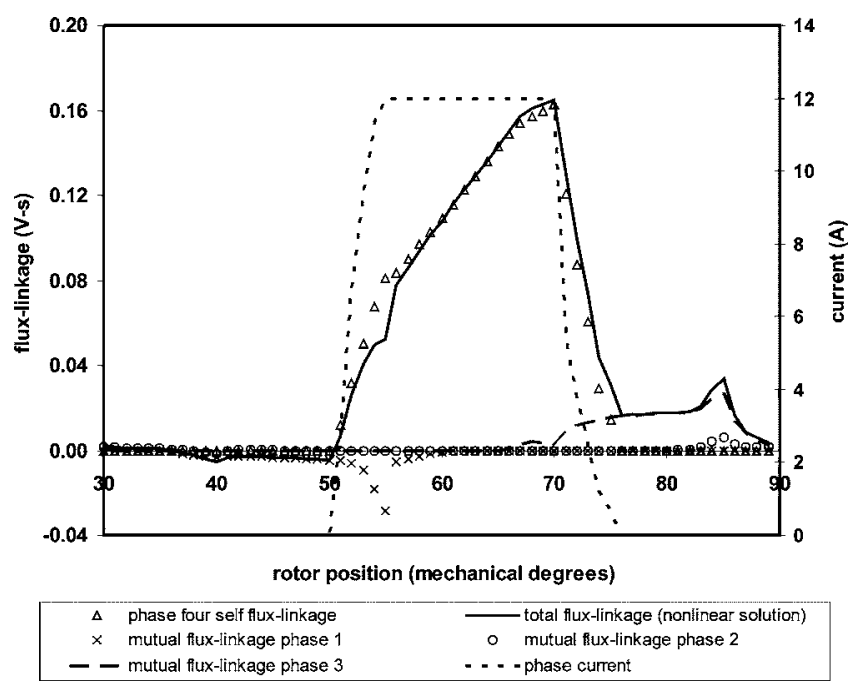

FIG. 5. Flux linkages of phase 4 from finite element solutions.

\section{DISCUSSION}

In the presented results, the mutual flux linkage created by the previous phase in the sequence is shown to be much smaller than that created by the next excited phase in the sequence (e.g., $M_{21} \ll M_{41}$ ), regardless of the phase polarities. The next phase in the sequence has the greatest effect on the shape of the $i-\psi$ loop and thus the torque produced.

To maximize the torque in phase 1 of the motor, the total flux linkage when the current is switched off should be positive. This cannot be achieved with balanced excitation wave forms, due to the negative mutual flux linkage from phase 4 . With certain control systems, it may be possible to excite the motor with different current wave forms in each phase. In such cases, the current wave forms of phases 4 and 1 can be modified to increase the total flux linkage during the overlap periods. There is a trade-off between reduction of the mutual flux linkage (due to phase 4) in phase 1 and reduction of the self-flux-linkage of phase 4 . Frozen permeability finite element simulations can be used to estimate the reduction in both the mutual flux linkage in phase 1 and self-flux-linkage in phase 4 .

In general, there are two courses of action that can be taken to reduce the effect of mutual flux linkage from the next phase in the sequence. Firstly, the turn-off angle of the current phase can be delayed, to ensure that the self-fluxlinkage of the phase is greater than the negative mutual flux linkage created by the next phase in the sequence, thus canceling out the mutual flux linkage. Secondly, the turn-on angle of current from the next phase in the sequence can be delayed or reduced.

\section{CONCLUSIONS}

This paper has shown the influence of mutual coupling on the per-phase magnetization characteristics of the switched reluctance motor. The magnitudes of the mutual flux linkages are shown to be strongly dependent on the polarity arrangement of the phases. The mutual coupling effects from each phase can be calculated using the finite element frozen permeability method. Further work shall investigate the finite element optimization of current excitation wave forms to reduce the influence of mutual coupling, using the frozen permeability method.

\section{ACKNOWLEDGMENTS}

The authors acknowledge the support of the SPEED Consortium. One of the authors (J. W.) is funded by the UK Engineering and Physical Sciences Research Consul, the SPEED Consortium, and Robert Bosch GmbH.

${ }^{1}$ T. J. E. Miller, Switched Reluctance Motors and Their Control (Magna Physics, Oxford, 1993).

${ }^{2}$ D. Panda and V. Ramanarayanan, IEEE Industry Applications Conference 1999, Phoenix, Arizona, 3-7 October 1999, Vol. 4, pp. 2227-2234.

${ }^{3}$ A. M. Michaelides and C. Pollock, IEE Proc.: Electr. Power Appl. 143, 361 (1996).

${ }^{4}$ N. Bianchi and S. Bolognani, IEEE Industry Applications Conference 1998, St. Louis, Missouri, 12-15 October 1998, Vol. 1, pp. 27-34.

${ }^{5}$ J. A. Walker, D. Dorrell, and C. Cossar, IEEE Trans. Magn. 41, 3946 (2005). 